\title{
Perspectives of Health Care Professionals' on delivering mHealth Sexual and Reproductive Health services in rural settings in Low-and- Middle-Income countries: a qualitative systematic review
}

\author{
Alexander Suuk Laar ( $\sim$ Alexander.Laar@uon.edu.au ) \\ The University of Newcastle \\ Melissa L. Harris \\ The University of Newcastle \\ Desalegn Markos Shifti \\ The University of Newcastle \\ Deborah Loxton \\ The University of Newcastle
}

\section{Research Article}

Keywords: Health care professionals, mobile phones, mHealth, Sexual and reproductive health, information and services, low-and middleincome countries

Posted Date: February 4th, 2022

DOI: https://doi.org/10.21203/rs.3.rs-1212113/v1

License: (1) This work is licensed under a Creative Commons Attribution 4.0 International License. Read Full License 


\section{Abstract}

Background: In low to middle income countries (LMICs) with limited health care providers (HCPs) and health infrastructure, digital technologies are rapidly being adopted to help augment service delivery. In this sphere, sexual and reproductive health (SRH) services are increasingly leveraging mobile health (mHealth) technologies to improve service and information provision in rural areas. This systematic review aimed to identify HCPs perspectives on barriers to, and facilitators of, mobile phone based SRH services and information in rural areas of LMICs from current literature.

Methods: Searches were conducted using the following databases: Medline, Scopus, PsychINFO, CINAHL and Cochrane Library. Based on the inclusion and exclusion criteria, twelve full text qualitative studies published in English between January 2000 and December 2020 were included. The methodological quality of papers was assessed by two authors using the critical skills appraisal programme and synthesized using the narrative thematic analysis approach.

Results: Positive HCPs experiences surrounding the provision of mHealth based SRH services in LMICs included saving consultation time, ability to shift tasks, reduction in travel costs, easy referrals and follow up on clients, convenience in communicating health information confidentially, and the ability to consult groups of clients remotely rather than face-to-face. Barriers to the provision of mHealth reported by HCPs included lack of technological infrastructure, unreliable networks, limited power, the cost of mobile airtime/data and mobile phones and limited technological literacy or skills.

Conclusions: Implementing innovative mHealth based SRH services could bridge a service provision and access gap of SRH information and services in rural areas of LMICs. Despite the advantages of this technology, several challenges associated with delivering $\mathrm{mHealth}$ SRH services need to be urgently addressed to enable scale-up and integration of sexual and reproductive mHealth into rural health systems.

\section{Background}

Low-and-middle-income countries (LMICs), where half of the world's population currently live [1], generally lack access to quality healthcare $[1,2]$. In many LMICs, variations in health may reflect the differences in the provision of, and access to, healthcare services and information [2]. In LMICs lack of complete access to health services is largely due to a lack of health care providers (HCPs) and health infrastructure [3, 4]. Rural areas of LMICs are consistently reported to have lower rates of HCPs and health facilities than urban areas [4, 5]. Some of the obstacles to SRH information and services in rural areas include a lack of health personnel, inadequate or unavailable healthcare facilities, long distances to healthcare facilities, poor transportation systems, and inability to afford the cost of health services [1, 3, 4]. A negative attitude among health providers and socio-cultural norms [6-8] also impact service access. These barriers result in an unmet need for SRH information and services, including contraception, which places women (and men) at increased risk of unintended pregnancy, sexually transmitted infections including HIV, and increased maternal morbidity and mortality [9-11].

One method being increasingly used in rural areas of LMICs to overcome some of these barriers is the delivery of health services via mobile phone, known as mobile health (mHealth) [12-14]. Evidence has shown several potential benefits for providing mHealth based SRH services in rural areas [14-20]. For example, reaching and engaging young people with a range of contraception information and services in the comfort of their locations or homes, as well as lowering the overall provision and access costs $[12,16,17,21,22]$. There is a growing evidence-base for the provision and use of mHealth interventions for SRH services and information in LMICs [16-19, 23-25]. The use of mHealth solutions for health system strengthening for SRH services in the rural context in LMICs is particularly attracting interest [17-19, $25,26]$.

Current evidence suggests that mHealth offers a promising method for provision of some SRH services to rural dwellers in LMICs $[9,14,17$, 24]. However, to appropriately inform policy before scaling up such services, a full understanding of the challenges and limitations of this method in the context of SRH is needed. The current study therefore reviewed existing literature where the experiences of HCPs in implementing mobile phone based SRH services in rural areas of LMICs had been explored. Specifically, we explored HCP experiences of barriers and facilitators in the delivery of mobile phone based SRH information and services to rural settings of LMICs.

\section{Methods}

\section{Protocol and registration}

This systematic review followed the preferred Reporting Items for Systematic Reviews and Meta-Analyses (PRISMA) guidelines [27]. It was registered with PROSPERO on October 23, 2020 (Prospero Number: CRD42020210777). 


\section{Database search}

We developed a search strategy for each database using the guidelines developed by the Cochrane Qualitative Research Methods Group for searching qualitative evidence [28]. A systematic search of six online journal databases was carried out to find relevant mHealth studies in the context of LMICs. Searches were limited to studies published in English from January 2000 to December 2020 as the field of mHealth has emerged over the last two decades [29].

Five domains were searched: "mHealth intervention provider", "mHealth platforms", "mHealth intervention recipient," "mHealth intervention services" and "geographical setting (LMICs)" (see Table 1).

Table 1

Search terms

\begin{tabular}{ll}
$\begin{array}{l}\text { Search } \\
\text { domains }\end{array}$ & Search Terms \\
$\begin{array}{l}\text { mHealth } \\
\text { intervention } \\
\text { provider }\end{array}$ & healthcare providers, lay health workers, health counsellors, healthcare workers, health educators \\
$\begin{array}{l}\text { mHealth } \\
\text { plattorms }\end{array}$ & $\begin{array}{l}\text { mobile health, mHealth, mobile phone health technology, mobile phone health, digital mobile health, digital mobile } \\
\text { phone health }\end{array}$ \\
$\begin{array}{l}\text { mHealth } \\
\text { intervention } \\
\text { recipient }\end{array}$ & $\begin{array}{l}\text { Women, men, adult men adult women, young, adolescent, young people, youth population, young women, young girls, } \\
\text { girls and boys }\end{array}$ \\
$\begin{array}{l}\text { mHealth } \\
\text { intervention } \\
\text { services }\end{array}$ & $\begin{array}{l}\text { reproductive health, sexual health, sexually transmitted infections such as HIV, contraception and family planning, } \\
\text { family planning information and services }\end{array}$ \\
\hline $\begin{array}{l}\text { Geographic } \\
\text { setting }\end{array}$ & \begin{tabular}{l} 
low-income countries, low-and-middle-income countries \\
\hline
\end{tabular}
\end{tabular}

Search terms

The first author (ASL) developed the search terms which were reviewed by MLH and DL. The search terms were then refined in consultation with the College of Health and Wellbeing's librarian. Search terms were combined with an "OR" Boolean operator, and terms between each domain were linked with "AND” operators.

\section{Data sources and search strategy}

We searched six databases (Medline, Scopus, PsychINFO, CINAHL and Cochrane Library) for published literature in English that reported on mHealth SRH intervention delivery barriers and facilitators for people in rural settings in LMICs. In addition to these sources, reference lists of all included studies and key references of relevant systematic reviews on mHealth studies available as well as Google were searched to identify any further relevant articles. The search terms which were used to perform Medline search strategy are shown in Table 2. Search strategies for the remaining databases are included in an online supplement.

Table 2

Medline Search Strategy

healthcare providers* OR Healthcare professionals* OR health provider* OR health counsellor* OR health educator* AND mobile health* OR mHealth* OR mobile phone health technology* OR mobile phone health* OR digital mobile health* OR digital mobile phone health* OR voice messaging*OR phone calls* OR voice calls* OR SMS text-messaging* OR short message service* OR IVR calls* OR interactive voice respose calls

AND

young adult* OR youth* OR adolescent* OR young people* OR youth population* OR young wom?n* OR young girl* OR young boy* OR young $m$ ? $n *$ OR young women* OR emerging adult* OR men* OR women* OR adult men* OR adult women* OR adolescent girl* OR adolescent boy* OR adolescen* AND reproductive health* or sexual health* or HIV* or contraception* or contraceptives* or modern contraception* or contracept service* or contracept educat* or contracept counsel*OR family planning

AND

low-income countries* OR low-and-middle-income nation* OR low to middle income countries* 0 middle-income countr* OR low resource countries

All limit to (english language and full text and humans and yr="2000 - 2020") 
The authors followed the 2009 Preferred Reporting Items for Systematic Review and Meta Analyses (PRISMA) flow chart [30] to report the study selection process (see Figure 1). The search results from the databases were first downloaded into the citation management system (Endnote X9 software)[31] and later imported into the Covidence online platform by the first author (ASL). Duplicates were automatically removed by the Covidence system. Data extraction to determine the relevancy of the papers was carried out by two authors (ASL and DMS). Following a data extraction form, the two authors independently read all included articles based on study author, year of publication, description of the study context, study methods, study population, mHealth intervention services, mHealth platforms and study findings. At all stages, any discrepancies were discussed until a consensus was reached.

\section{Inclusion and exclusion criteria}

We included studies that reported on mHealth interventions which reported information on SRH information for people in rural settings in LMICs according to World Bank classifications [32]. Studies not peer-reviewed for example, conference presentations, student theses, editorials, review articles, letters to the editor, commentaries, and symposium proceedings were excluded.

\section{Results}

\section{Study selection}

Two authors (ASL and DMS) independently reviewed the full text articles for suitability for the review. A total of 92 full text articles were assessed according to the selection criteria and 12 studies were retained for qualitative synthesis (Fig. 1).

\section{Quality Assessment}

\section{Critical Appraisal of included studies}

Two authors (ASL and DMS) independently and critically appraised 12 eligible papers for methodological quality using critical appraisal tool for mixed studies review (MSR) [33]. We appraised the studies in line with the presence or absence of a primary qualitative study questions, study design, sampling method, study context, data collection, data analysis, ethical considerations, researchers' reflexivity, conclusions drawn justified by study findings, transferability of study findings to similar settings (Table 3 ). The methodological quality of all included studies were assessed based on a ten point question criteria. For each criterion, the presence denoted yes scored as 1 and absence no scored as 0 respectively. The studies were scored using percentages (0-100\% with one point representing $10 \%)$. The scores ranged from $50-100 \%$. They were interpreted as follows: below 50\% low quality, $50-75 \%$ average quality, and $76-100 \%$ high quality (Table 3 ). The quality score was calculated as [(number of yes responses divided by the number of the relevant criteria (10) x100]. Based on the scoring system, we retained all 12 primary studies for the review. 
Table 3

MSR quality appraisal procedures

\begin{tabular}{|c|c|c|c|c|c|c|c|c|c|c|c|}
\hline \multirow[t]{2}{*}{ Study Authors } & \multicolumn{11}{|c|}{ Quality assessment questions } \\
\hline & 1 & 2 & 3 & 4 & 5 & 6 & 7 & 8 & 9 & 10 & Total (n\%) \\
\hline Jahangir et al [34] & Yes & Yes & Yes & Yes & Yes & Yes & Yes & No & Yes & Yes & $9(90 \%)$ \\
\hline Peprah et al [35] & Yes & Yes & Yes & Yes & Yes & Yes & Yes & Yes & Yes & Yes & $10(100 \%)$ \\
\hline Braun et al [36] & Yes & Yes & Yes & Yes & Yes & No & Yes & No & Yes & Yes & $8(80 \%$ \\
\hline Dev et al [37] & Yes & Yes & Yes & Yes & Yes & Yes & Yes & Yes & Yes & Yes & $10(100 \%)$ \\
\hline Logle et al [38] & Yes & Yes & No & yes & No & No & No & No & Yes & Yes & $50(50 \%)$ \\
\hline Ibembe, [39] & Yes & Yes & Yes & Yes & Yes & Yes & Yes & No & Yes & Yes & $9(90 \%)$ \\
\hline Ong et al [40] & Yes & Yes & Yes & Yes & Yes & Yes & Yes & No & Yes & Yes & $9(90 \%)$ \\
\hline Khatun et al [41] & Yes & Yes & Yes & Yes & Yes & Yes & No & No & Yes & Yes & $8(80 \%)$ \\
\hline Hirsch-Moverman et al [42] & Yes & Yes & Yes & Yes & Yes & Yes & Yes & Yes & Yes & Yes & $10(100 \%)$ \\
\hline Jennings et al [25] & Yes & Yes & Yes & Yes & Yes & Yes & Yes & No & Yes & Yes & $9(90 \%)$ \\
\hline Hampshere et al [43] & Yes & Yes & Yes & Yes & Yes & Yes & Yes & Yes & Yes & Yes & $10(100)$ \\
\hline Chang et al [44] & Yes & Yes & Yes & Yes & Yes & Yes & Yes & No & Yes & Yes & $9(90 \%)$ \\
\hline \multicolumn{12}{|l|}{ Total=12 } \\
\hline \multicolumn{12}{|l|}{ Key } \\
\hline \multicolumn{5}{|c|}{$\begin{array}{l}\text { 1. Were the objective(s) or question(s) of the research clearly } \\
\text { stated? } \\
\text { 2. Was a qualitative approach appropriate for the research } \\
\text { question? }\end{array}$} & 6. Wo & he da & analys & ely ac & ss pot & bed? & scribed? \\
\hline \multicolumn{5}{|c|}{ 3. Was the sampling strategy used appropriate and described? } & \multicolumn{7}{|c|}{ 8. Does the study adequately address reflexivity issues? } \\
\hline \multicolumn{5}{|c|}{ 4. Was the study context clearly described? } & \multicolumn{7}{|c|}{ 9. Were the conclusions drawn justified by the findings? } \\
\hline & & & & & \multicolumn{7}{|c|}{$\begin{array}{l}\text { 10. Are the findings of the study transferable to my own and other } \\
\text { settings? }\end{array}$} \\
\hline
\end{tabular}

\section{Characteristics of included studies}

Of the 12 studies meeting the inclusion criteria, ten were conducted in rural areas [25, 34-39, 41, 42, 44], one in rural/urban areas [40] and one rural and peri-urban areas [43] respectively. All the included studies provided evidence on mHealth SRH information and services [25, 34-44]. The included studies were conducted in the following countries: two in Bangladesh [34, 41], three in Kenya [25, 37, 39], one in Ghana [35], one in Ghana and Malawi [43], one in Tanzania [36], one in Lesotho [42], one in Nigeria and Kenya [38], one in Uganda [44] and one in Cambodia [40]. Most studies (9) used qualitative method designs [25, 34-37, 39, 40,42, 44] with only three using mixed methods designs $[38,41,43]$.These studies involved male and female populations in community and health facility settings. All studies reported HCPs experiences on facilitators and barriers for delivering mobile phone based reproductive health services [25, 34-44]. Data was analysed thematically (Table 4). 
Table 4

Summary of Studies included in the Systematic Review, N=12

\begin{tabular}{|c|c|c|c|c|c|c|}
\hline $\begin{array}{l}\text { Author \& } \\
\text { year }\end{array}$ & $\begin{array}{l}\text { Country\& } \\
\text { Setting }\end{array}$ & $\begin{array}{l}\text { Study } \\
\text { methods }\end{array}$ & $\begin{array}{l}\text { Study } \\
\text { population }\end{array}$ & $\begin{array}{l}\text { mHealth } \\
\text { interventionchannels/platforms }\end{array}$ & Barriers & Facilitators \\
\hline $\begin{array}{l}\text { Jahangir } \\
\text { et al [34] }\end{array}$ & $\begin{array}{l}\text { Bangladesh } \\
\text { Community- } \\
\text { based } \\
\text { Rural }\end{array}$ & $\begin{array}{l}\text { Qualitative } \\
\text { In-depth } \\
\text { interviews }\end{array}$ & $\begin{array}{l}\text { Health } \\
\text { providers }\end{array}$ & $\begin{array}{l}\text { Sexual health services } \\
\text { SMS text-messaging }\end{array}$ & $\begin{array}{l}\text { - Low levels of } \\
\text { technological } \\
\text { literacy. } \\
\text {-Diagnoses of } \\
\text { STIs over the } \\
\text { phone not } \\
\text { always possible } \\
\text { due to physical } \\
\text { examination. } \\
\text {-High calls and } \\
\text { time } \\
\text { management is } \\
\text { a difficult task } \\
\text { at times. }\end{array}$ & $\begin{array}{l}\text {-mHealth } \\
\text { platform } \\
\text { services quite } \\
\text { good for } \\
\text { providing } \\
\text { counselling. } \\
\text {-Gets quick } \\
\text { information to } \\
\text { clients } \\
\text { - Easy referral } \\
\text { services by } \\
\text { providing } \\
\text { phone numbers } \\
\text { for nearby } \\
\text { clinics or } \\
\text { diagnostic } \\
\text { centers for } \\
\text { clients. } \\
\text { - Easy way to } \\
\text { give valuable } \\
\text { and timely } \\
\text { information to } \\
\text { the client } \\
\text {-mHealth is } \\
\text { effective way in } \\
\text { providing } \\
\text { greater access } \\
\text { to health } \\
\text { information. }\end{array}$ \\
\hline $\begin{array}{l}\text { Peprah et } \\
\text { al [35] }\end{array}$ & $\begin{array}{l}\text { Ghana } \\
\text { Rural }\end{array}$ & $\begin{array}{l}\text { Qualitative } \\
\text { In-depth } \\
\text { interviews }\end{array}$ & $\begin{array}{l}\text { Healthcare } \\
\text { providers }\end{array}$ & $\begin{array}{l}\text { Sexual and reproductive } \\
\text { services } \\
\text { Phone call }\end{array}$ & $\begin{array}{l}\text {-Language } \\
\text { barrier. } \\
\text {-llliteracy of } \\
\text { recipients. } \\
\text { - Lack of trust. } \\
\text {-Mobile network } \\
\text { connectivity } \\
\text { challenges. }\end{array}$ & $\begin{array}{l}\text { mHealth saves } \\
\text { waiting hours' } \\
\text { time. } \\
\text {-Delivering } \\
\text { services via } \\
\text { mHealth } \\
\text { technology } \\
\text { saves time. } \\
\text {-mHealth } \\
\text { reduces } \\
\text { workload. } \\
\text {-mHealth able } \\
\text { to contact } \\
\text { many clients at } \\
\text { a time for } \\
\text { healthcare. }\end{array}$ \\
\hline
\end{tabular}




\begin{tabular}{|c|c|c|c|c|c|c|}
\hline $\begin{array}{l}\text { Author \& } \\
\text { year }\end{array}$ & $\begin{array}{l}\text { Country\& } \\
\text { Setting }\end{array}$ & $\begin{array}{l}\text { Study } \\
\text { methods }\end{array}$ & $\begin{array}{l}\text { Study } \\
\text { population }\end{array}$ & $\begin{array}{l}\text { mHealth } \\
\text { interventionchannels/platforms }\end{array}$ & Barriers & Facilitators \\
\hline \multirow{7}{*}{$\begin{array}{l}\text { Braun et al } \\
\text { [36] }\end{array}$} & Tanzania & Qualitative & \multirow{7}{*}{$\begin{array}{l}\text { Community } \\
\text { health } \\
\text { workers }\end{array}$} & Family planning (FP) services & \multirow{3}{*}{$\begin{array}{l}\text {-Low } \\
\text { technological } \\
\text { skills. } \\
\text {-Limited power } \\
\text { for battery } \\
\text { charging }\end{array}$} & \multirow{2}{*}{$\begin{array}{l}\text { - Timelier } \\
\text { provision of FP } \\
\text { care and } \\
\text { services. }\end{array}$} \\
\hline & \multirow[t]{6}{*}{ Rural } & \multirow[t]{6}{*}{ IDIs } & & SMS text-messaging & & \\
\hline & & & & & & \multirow{2}{*}{$\begin{array}{l}\text { - Convenient } \\
\text { contacting } \\
\text { clients from } \\
\text { various } \\
\text { locations. And } \\
\text { providing care. }\end{array}$} \\
\hline & & & & & \multirow[t]{4}{*}{$\begin{array}{l}\text {-Cost of mobile } \\
\text { phone. }\end{array}$} & \\
\hline & & & & & & $\begin{array}{l}\text {-Ease of use of } \\
\text { technology }\end{array}$ \\
\hline & & & & & & $\begin{array}{l}\text { confidential } \\
\text { information } \\
\text { and care. }\end{array}$ \\
\hline & & & & & & $\begin{array}{l}\text {-Convenient to } \\
\text { provide } \\
\text { services at } \\
\text { anytime and } \\
\text { anywhere. }\end{array}$ \\
\hline \multirow{7}{*}{$\begin{array}{l}\text { Dev et al } \\
\text { [37] }\end{array}$} & Kenya & Qualitative & \multirow{7}{*}{$\begin{array}{l}\text { Healthcare } \\
\text { providers } \\
\text { (nurses) }\end{array}$} & Contraception services & \multirow{7}{*}{$\begin{array}{l}\text { - Technological } \\
\text { literacy } \\
\text { challenge. } \\
\text { - Workload and } \\
\text { time constraints } \\
\text { meeting mobile } \\
\text { phone services } \\
\text { demand by } \\
\text { clients. } \\
\text {-Heavy workload } \\
\text { for receiving } \\
\text { calls and } \\
\text { messages } \\
\text { leaving little } \\
\text { time to provide } \\
\text { FP counselling } \\
\text { services. }\end{array}$} & \multirow{3}{*}{$\begin{array}{l}\text {-Helps deliver } \\
\text { the appropriate } \\
\text { and detailed } \\
\text { information on } \\
\text { FP methods. }\end{array}$} \\
\hline & \multirow[t]{6}{*}{ Rural } & \multirow[t]{6}{*}{$\begin{array}{l}\text { In-depth } \\
\text { interviews }\end{array}$} & & \multirow[t]{6}{*}{ Mobile phone call } & & \\
\hline & & & & & & \\
\hline & & & & & & $\begin{array}{l}\text {-Reduces or } \\
\text { saves time to } \\
\text { provide } \\
\text { comprehensive } \\
\text { counselling. }\end{array}$ \\
\hline & & & & & & $\begin{array}{l}\text {-Improves client } \\
\text { provider } \\
\text { interactions. }\end{array}$ \\
\hline & & & & & & $\begin{array}{l}\text { - Allows } \\
\text { discuss } \\
\text { confidential } \\
\text { issues with } \\
\text { women on } \\
\text { contraceptives } \\
\text { privately with } \\
\text { women to } \\
\text { make better } \\
\text { decisions }\end{array}$ \\
\hline & & & & & & $\begin{array}{l}\text {-Maximizes } \\
\text { time or saves } \\
\text { providers time } \\
\text { in providing } \\
\text { counselling } \\
\text { process. }\end{array}$ \\
\hline
\end{tabular}




\begin{tabular}{|c|c|c|c|c|c|c|}
\hline $\begin{array}{l}\text { Author \& } \\
\text { year }\end{array}$ & $\begin{array}{l}\text { Country\& } \\
\text { Setting }\end{array}$ & $\begin{array}{l}\text { Study } \\
\text { methods }\end{array}$ & $\begin{array}{l}\text { Study } \\
\text { population }\end{array}$ & $\begin{array}{l}\text { mHealth } \\
\text { interventionchannels/platforms }\end{array}$ & Barriers & Facilitators \\
\hline $\begin{array}{l}\text { Logie et al } \\
\text { [38] }\end{array}$ & $\begin{array}{l}\text { Nigeria } \\
\text { Keyna } \\
\text { Rural }\end{array}$ & $\begin{array}{l}\text { Mixed method } \\
\text { Qualitive In- } \\
\text { depth } \\
\text { interviews } \\
\text { (IDIs) }\end{array}$ & $\begin{array}{l}\text { Healthcare } \\
\text { providers }\end{array}$ & $\begin{array}{l}\text { Sexual and reproductive health } \\
\text { services } \\
\text { Mobile phone call }\end{array}$ & $\begin{array}{l}\text {-Does not work } \\
\text { if a client/ } \\
\text { person doesn't } \\
\text { have a phone. } \\
\text {-Lack of regular } \\
\text { internet access. } \\
\text {-Lack of } \\
\text { technological } \\
\text { literacy of using } \\
\text { mobile apps. } \\
\text {-Poor } \\
\text { populations } \\
\text { struggle to use } \\
\text { phones due to } \\
\text { cost. }\end{array}$ & $\begin{array}{l}\text {-Able to target } \\
\text { specific health } \\
\text { information to } \\
\text { clients in rural } \\
\text { areas based on } \\
\text { health } \\
\text { demographics. } \\
\text {-Able to provide } \\
\text { access to SRH } \\
\text { information for } \\
\text { underserved } \\
\text { group. } \\
\text { - Easier to } \\
\text { provide } \\
\text { mHealth apps } \\
\text { SRH for young } \\
\text { people in } \\
\text { remote areas. } \\
\text {-Easy for } \\
\text { healthcare } \\
\text { providers to } \\
\text { constantly } \\
\text { remind or } \\
\text { follow up on } \\
\text { clients or } \\
\text { patient. }\end{array}$ \\
\hline $\begin{array}{l}\text { lbembe } \\
\text { [39] }\end{array}$ & $\begin{array}{l}\text { Kenya } \\
\text { Health } \\
\text { facility } \\
\text { Rural }\end{array}$ & $\begin{array}{l}\text { Qualitative } \\
\text { IDIs }\end{array}$ & $\begin{array}{l}\text { Healthcare } \\
\text { professionals }\end{array}$ & $\begin{array}{l}\text { Reproductive health services } \\
\text { Mobile phone call }\end{array}$ & $\begin{array}{l}\text {-Lack of ICT } \\
\text { support systems } \\
\text { and expertise at } \\
\text { the local levels. } \\
\text {-Poor network } \\
\text { connectivity. } \\
\text {-Cost of phone } \\
\text { credit or airtime. } \\
\text {-Lack of } \\
\text { motivation. } \\
\text {-Lack of } \\
\text { technological } \\
\text { literacy and } \\
\text { skills. } \\
\text { - Not owning a } \\
\text { cell phone. }\end{array}$ & $\begin{array}{l}\text {-Easy } \\
\text { consultation. } \\
\text { - Addressing } \\
\text { challenges } \\
\text { distance } \\
\text { between health } \\
\text { providers and } \\
\text { users. } \\
\text { - Trust and } \\
\text { confidentiality } \\
\text { are built. } \\
\text { around health } \\
\text { providers and } \\
\text { users. } \\
\text {-Quality and } \\
\text { timely decision } \\
\text { making } \\
\text { between client } \\
\text { and medical } \\
\text { personnel. }\end{array}$ \\
\hline
\end{tabular}




\begin{tabular}{|c|c|c|c|c|c|c|}
\hline $\begin{array}{l}\text { Author \& } \\
\text { year }\end{array}$ & $\begin{array}{l}\text { Country\& } \\
\text { Setting }\end{array}$ & $\begin{array}{l}\text { Study } \\
\text { methods }\end{array}$ & $\begin{array}{l}\text { Study } \\
\text { population }\end{array}$ & $\begin{array}{l}\text { mHealth } \\
\text { interventionchannels/platforms }\end{array}$ & Barriers & Facilitators \\
\hline $\begin{array}{l}\text { Ong et al } \\
{[40]}\end{array}$ & $\begin{array}{l}\text { Cambodia } \\
\text { Rural/urban }\end{array}$ & $\begin{array}{l}\text { Qualitative } \\
\text { Focus group } \\
\text { discussions } \\
\text { (FGDs) IDIs }\end{array}$ & $\begin{array}{l}\text { Community } \\
\text { health } \\
\text { workers }\end{array}$ & $\begin{array}{l}\text { Sexual and reproductive } \\
\text { health/HIV } \\
\text { SMS text-messaging } \\
\text { Voice Messaging }\end{array}$ & $\begin{array}{l}\text {-Lack of } \\
\text { financial } \\
\text { support for } \\
\text { service } \\
\text { provision. } \\
\text {-Network } \\
\text { connectivity } \\
\text { interruptions. }\end{array}$ & $\begin{array}{l}\text { - Able to } \\
\text { provide } \\
\text { information on } \\
\text { HIV and STIs } \\
\text { prevention } \\
\text { issues. } \\
\text { - Able to link up } \\
\text { with many } \\
\text { clients with } \\
\text { SRH services. } \\
\text {-Able to } \\
\text { connect clients } \\
\text { to the nearest } \\
\text { relevant health } \\
\text { facility. } \\
\text {-Able to help } \\
\text { clients in } \\
\text { making } \\
\text { decisions } \\
\text { about } \\
\text { professional } \\
\text { assistance. } \\
\text {-More efficient } \\
\text { to deliver } \\
\text { information } \\
\text { directly and } \\
\text { more frequently } \\
\text { to a larger } \\
\text { group via } \\
\text { mobile phones. }\end{array}$ \\
\hline $\begin{array}{l}\text { Khatun et } \\
\text { al [41] }\end{array}$ & $\begin{array}{l}\text { Bangladesh } \\
\text { Rural }\end{array}$ & $\begin{array}{l}\text { Mixed method } \\
\text { QualitativelDIs }\end{array}$ & & $\begin{array}{l}\text { Health services } \\
\text { Mobile phone call }\end{array}$ & $\begin{array}{l}\text {-Technological } \\
\text { human resource } \\
\text { inadequacy. } \\
\text {-Technological } \\
\text { readiness by } \\
\text { facilities and } \\
\text { healthcare } \\
\text { personnel. } \\
\text {-Community } \\
\text { members lack } \\
\text { of technological } \\
\text { skills. } \\
\text {-Healthcare } \\
\text { professionals } \\
\text { lack of } \\
\text { technological } \\
\text { skills. } \\
\text { - Community } \\
\text { members lack } \\
\text { of English } \\
\text { language } \\
\text { proficiency and } \\
\text { illiteracy } \\
\text { barriers. }\end{array}$ & $\begin{array}{l}\text {-Decrease in } \\
\text { patient loads in } \\
\text { rural healthcare } \\
\text { centers. } \\
\text {-mHealth } \\
\text { consultation } \\
\text { saves time } \\
\text { - Enables } \\
\text { health } \\
\text { providers to } \\
\text { provide quality } \\
\text { health services. }\end{array}$ \\
\hline
\end{tabular}




\begin{tabular}{|c|c|c|c|c|c|c|}
\hline $\begin{array}{l}\text { Author \& } \\
\text { year }\end{array}$ & $\begin{array}{l}\text { Country\& } \\
\text { Setting }\end{array}$ & $\begin{array}{l}\text { Study } \\
\text { methods }\end{array}$ & $\begin{array}{l}\text { Study } \\
\text { population }\end{array}$ & $\begin{array}{l}\text { mHealth } \\
\text { interventionchannels/platforms }\end{array}$ & Barriers & Facilitators \\
\hline $\begin{array}{l}\text { Hirsch- } \\
\text { Moverman } \\
\text { et al [42] }\end{array}$ & $\begin{array}{l}\text { Lesotho } \\
\text { Rural }\end{array}$ & $\begin{array}{l}\text { Qualitative } \\
\text { IDIs }\end{array}$ & $\begin{array}{l}\text { Healthcare } \\
\text { providers } \\
\text { Health } \\
\text { facility \& } \\
\text { community - } \\
\text { based }\end{array}$ & $\begin{array}{l}\text { Health services/HIV } \\
\text { SMS text-messaging }\end{array}$ & $\begin{array}{l}\text {-Lack of } \\
\text { technology } \\
\text { infrastructure. } \\
\text {-Network } \\
\text { connectivity } \\
\text { challenges. } \\
\text {-Limited } \\
\text { electricity } \\
\text { connectivity. }\end{array}$ & $\begin{array}{l}\text {-Facilitates } \\
\text { communication } \\
\text { between } \\
\text { patients' } \\
\text { providers. } \\
\text {-Ability to be } \\
\text { able to follow- } \\
\text { up patients } \\
\text { frequently. } \\
\text {-mHealth } \\
\text { communication } \\
\text { messages } \\
\text { strengthen the } \\
\text { patient- } \\
\text { provider bond. } \\
\text {-Ability to } \\
\text { monitor } \\
\text { patients over } \\
\text { phone. } \\
\text {-Easily able to } \\
\text { track and } \\
\text { follow up } \\
\text { patients. }\end{array}$ \\
\hline $\begin{array}{l}\text { Jennings } \\
\text { et al [25] }\end{array}$ & $\begin{array}{l}\text { Kenya } \\
\text { Rural }\end{array}$ & FGDs/IDIs & $\begin{array}{l}\text { Community } \\
\text { health } \\
\text { workers \& } \\
\text { nurses } \\
\text { Health } \\
\text { facility-based }\end{array}$ & $\begin{array}{l}\text { HIV services } \\
\text { Voice calls, } \\
\text { SMS text-messaging }\end{array}$ & $\begin{array}{l}\text { - Purchasing } \\
\text { airtime for } \\
\text { phones. } \\
\text {-Not able to } \\
\text { ascertain } \\
\text { whether. clients } \\
\text { are really } \\
\text { following the } \\
\text { advice or } \\
\text { counselling } \\
\text { given to them. }\end{array}$ & $\begin{array}{l}\text { - Protects the } \\
\text { confidentiality } \\
\text { of information. } \\
\text {-Helpful using } \\
\text { mobile phones } \\
\text { to follow up on } \\
\text { patients } \\
\text { through called } \\
\text { or sent SMS } \\
\text { texts to them. } \\
\text {-Easy referral } \\
\text { and follow up } \\
\text { on clients to } \\
\text { other HCPs. } \\
\text {-Helps to } \\
\text { quickly notify } \\
\text { clients about } \\
\text { appointments } \\
\text { or to } \\
\text { reschedule } \\
\text { client visits to } \\
\text { save them time } \\
\text { and } \\
\text { unnecessary } \\
\text { visits. }\end{array}$ \\
\hline
\end{tabular}




\begin{tabular}{|c|c|c|c|c|c|c|}
\hline $\begin{array}{l}\text { Author \& } \\
\text { year }\end{array}$ & $\begin{array}{l}\text { Country\& } \\
\text { Setting }\end{array}$ & $\begin{array}{l}\text { Study } \\
\text { methods }\end{array}$ & $\begin{array}{l}\text { Study } \\
\text { population }\end{array}$ & $\begin{array}{l}\text { mHealth } \\
\text { interventionchannels/platforms }\end{array}$ & Barriers & Facilitators \\
\hline $\begin{array}{l}\text { Hampshire } \\
\text { et al [43] }\end{array}$ & $\begin{array}{l}\text { Ghana \& } \\
\text { Malawi } \\
\text { Peri-urban \& } \\
\text { rural }\end{array}$ & $\begin{array}{l}\text { Mixed method } \\
\text { Qualitative } \\
\text { IDIs }\end{array}$ & $\begin{array}{l}\text { Health } \\
\text { workers } \\
\text { Community- } \\
\text { based }\end{array}$ & $\begin{array}{l}\text { Contraception/family } \\
\text { planning/HIV services } \\
\text { SMS text-messaging } \\
\text { Phone calls } \\
\text { Voice messages }\end{array}$ & $\begin{array}{l}\text {-Not having } \\
\text { personal mobile } \\
\text { phones. } \\
\text {-Temporary } \\
\text { mobile phone } \\
\text { breakdown can } \\
\text { be problematic. } \\
\text {-Poor or } \\
\text { unreliable } \\
\text { network } \\
\text { - Mobile phone } \\
\text { credit or airtime. } \\
\text {-Limited sources } \\
\text { to buy credit } \\
\text { Emotional } \\
\text { burden for } \\
\text { receiving calls } \\
\text { at night. }\end{array}$ & $\begin{array}{l}\text {-Mobile } \\
\text { phones help in } \\
\text { emergencies, } \\
\text { staff making } \\
\text { emergency } \\
\text { calls. } \\
\text {-Helps in } \\
\text { communicating } \\
\text { with patients' } \\
\text { colleagues, } \\
\text { obtaining } \\
\text { clinical advice. }\end{array}$ \\
\hline $\begin{array}{l}\text { Chang et } \\
\text { al [43] }\end{array}$ & $\begin{array}{l}\text { Uganda } \\
\text { Rural }\end{array}$ & $\begin{array}{l}\text { Qualitative } \\
\text { IDIs }\end{array}$ & $\begin{array}{l}\text { Peer health } \\
\text { workers }\end{array}$ & $\begin{array}{l}\text { HIV services } \\
\text { SMS text-messaging } \\
\text { Voice messaging }\end{array}$ & $\begin{array}{l}\text {-Phone } \\
\text { maintenance } \\
\text { cost } \\
\text {-Lack of } \\
\text { power/electricity } \\
\text { to charge } \\
\text { phones. } \\
\text {-Mobile phones } \\
\text { theft. }\end{array}$ & $\begin{array}{l}\text {-Task shifting. } \\
\text { Time saving. } \\
\text {-Facilitates } \\
\text { exchange of } \\
\text { information or } \\
\text { communication } \\
\text { between HCPs } \\
\text { and patients } \\
\text { and HCPs. } \\
\text {-lmproved peer } \\
\text { health workers } \\
\text { morale. }\end{array}$ \\
\hline
\end{tabular}

mHealth intervention services delivered by HCPs

In this review, all the mHealth intervention services for SRH information and services involved contraception, family planning and HIV prevention education among people in rural areas in LMICs [25, 34-44].

HCPs experiences for the provision of mHealth-based SRH services

\section{Facilitators to mHealth SRH services provision}

The review findings have provided insights into HCPs views and experiences on factors acting as facilitators for the provision of mHealthbased SRH services for people in rural areas of LMICs [25, 34-44]. Most HCPs were supportive of the mHealth application for helping to address some of the challenges of providing SRH information and services in rural areas. Participants reported that mobile phone technology helps make communication of SRH information and services to hard-to-reach rural areas remotely [34] confidentially compared to face-to-face consultation [25, 35-37, 39].

Advantages of mHealth technology also provided time efficiency because multiple health information and services could be delivered to groups of people $[25,40,44]$. There were also cost savings for both HCPs and clients because there was no need to travel to health facilities [43]. In addition, HCPs said using mobile phones made it possible to task shift some responsibilities to lower cadre of health workforce remotely $[25,36,37,40,43]$. HCPs also described that using the mobile phone technology helped facilitate referrals and follow up on clients to HCPs in health facilities.

\section{Barriers to mHealth SRH services provision}

Factors reported by HCPs for acting as barriers for the provision of mHealth-based SRH services for people mainly consisted of technological challenges including limited and unreliable network connectivity [38-40,42, 43], limited power for charging mobile phones $[36,42,44]$, cost of mobile phones, charging and maintenance [36, 38, 39], limited vendors or outlets for sale of mobile credit or airtime [43], technological literacy skills [35, 39, 41,42], cost for mobile credit or airtime and affordability prevented some calls to be made [35, 39, 43] since such expenses were not covered by mHealth intervention programs. HCPs also reported lack of technological infrastructure [39, 41 , 
42] for delivering and accessing services [34-39, 41], and emotional burden and workload for attending to clients calls [34, 37, 43]. HCPs said these challenges hindered effective provision of mHealth $\mathrm{SRH}$ services.

\section{Discussion}

Mobile health interventions have the potential to improve the provision of SRH services in LMICs $[16,18,20,45,46]$ by connecting directly to people in rural areas to engage with HCPs $[16,18,20,45,46]$. Rural settings in LMICs may pose both significant opportunities and challenges for mHealth [47]. This review provides evidence on facilitators and barriers for delivering mobile phone based SRH information and services to people in rural settings in LMICs experienced by HCPs [25, 34-44].

Our findings showed that HCPs perceived mHealth to be useful for providing SRH services to rural people [43]. Study participants reported facilitators such as the convenience of using mobile phone to deliver a range of SRH information and services remotely and confidentially $[25,34-44]$ reducing fear and stigma associated with face-to-face SRH consultations. Also, saving of travel time and costs for both HCPs and users were noted [36, 43, 44], in line with research [6, 48-50].

An important facilitator for providing mHealth was the ability to task shift by delegating duties or responsibilities to lower-level cadre health professionals $[34,44]$. HCPs said task shifting helped improved time management and workload for them to perform critical and urgent duties $[25,36,37,40,43]$. Task shifting has been identified as a pragmatic response to health workforce shortages in rural settings in LMICs [51]. It is observed however that the burden of task shifting tends to fall disproportionally on HCPs with lower qualifications and volunteers, leading to work overload without corresponding remuneration [51,52]. To maximize task shifting benefits without placing an undue burden on HCPs who are willing to undertake additional workload, appropriate compensation and training need to be considered, to ensure the sustainability of mHealth programs in rural settings in LMICs [43].

In this review, services were provided using voice messaging, phone calls, voice calls and SMS text-messaging [25, 34-44]. SMS texting was seen as the most preferred and efficient option for delivering health information and services, due to the ability to transmit multiple health messages to groups of people at the same time and confidentially [34-36, 42]. A preference for delivering health information via SMS text messages in rural populations in LMICs settings has been reported [53]. There is a growing interest for the preference of mHealth interventions platforms in LMICs for SRH information and services for rural population. There is the need for research to understand the benefits and preferences of mobile phone-based platforms for users with greater reach in rural areas especially among lower literate populations.

The review also highlighted technological challenges which hindered the effective delivery of SRH mHealth services [25, 34-44].The major barriers included a lack of technical skills [35, 39, 41, 42] and limited technological infrastructure [39, 41, 42]. These findings have been reported by studies in LMICs $[18,45,54,55]$. The full realization of the full potential of mHealth SRH services will require investment in the development of technological infrastructure $[35,56]$ and building the capacity of HCPs to effectively advance mHealth SRH services for rural populations $[17,57,58]$.

In this review, HCPs also reported personal and contextual challenges such as cost of mobile phones and lack of electricity for charging mobile phones [36, 38, 39], cost for mobile credit/ airtime [35, 39, 43] and unreliable or weak network connectivity [35, 39, 40, 42]. Several studies conducted in similar settings in LMICs have confirmed these findings $[16,18,45,59,60]$. In some instances, HCPs had to bear mobile phone expenses in order to be able to provide the services [43]. A qualitative study in rural South Africa has reported similar findings [12]. Personal cost of providing health delivery services in rural settings in LMICs constitutes a disproportionate share of HCPs already low incomes [43]. Subsidizing mHealth service provision for rural health workers in LMICs is critical for delivery and use of SRH services in rural health systems $[15,61,62]$. Contextual barriers [39, 41, 42] including lack or unreliable network connectivity [38-40, 42, 43], lack of electricity to charge mobile phones $[36,42,44]$ and outlets for airtime retailers [43] influence HCPs ability to effective delivery of services to rural populations.

Although mHealth programs are becoming an integral part of SRH services in rural LMICs [20,46], investment in the interventions need to be complemented by a thorough evaluation of contextual factors to effectively address provider and user needs for improvement of health outcomes. The review findings provide programme managers and policy makers with evidence to suggest that addressing economic and infrastructure gaps for providing mHealth SRH services in rural settings in LMICs will require a collaboration between governments, nongovernmental organizations and other stakeholders.

\section{Strengths And Limitations}


A strength of this study is that it gives a clear review of the practical experiences of HCPs on facilitators and challenges for providing mHealth SRH services in rural settings in LMICs. Another strength of this study is that it covered a period of two decades from the inception of mHealth to date. In addition, all primary studies included in this review underwent a rigorous methodological quality appraisal. A major limitation of this study is that only studies written and published in English were included.

\section{Conclusions}

There have been few studies of mHealth on barriers and facilitators for improving population health in rural settings in LMICs. Our review found that implementing innovative mHealth based SRH services could bridge a service access gap of SRH information and services in rural areas of LMICs. Despite the advantages of this technology, several challenges associated with delivering mHealth need to be urgently addressed to enable scale-up and integration of sexual and reproductive mHealth into rural health systems. Our recommendations serve as references for improving on existing mHealth services and the implementation of future studies in rural LMICs. However, further research is needed to explore HCPs experiences on the effectiveness of using mobile phone communication platforms for delivering SRH information and services in rural settings in LMICs. Furthermore, it is likely that mHealth service barriers and facilitators vary by cultural and country setting, underscoring the need for more nuanced research in this area.

\section{Declarations}

\section{Ethics approval and consent to participate}

Not applicable.

\section{Consent for publication}

Not applicable

\section{Availability of data and materials}

The datasets used and/or analysed during the current study are available from the corresponding author on reasonable request.

\section{Competing interest}

None declared.

\section{Funding}

This study was not funded by The University of Newcastle, Australia

\section{Acknowledments}

We would like to acknowledge Jessica Birchall, senior librarian at the University of Newcastle for her guidance in developing the search terms for this review. Dr Melissa Harris is supported by an ARC Discovery Early Career Researcher Award ((DE190101134).

\section{Authors' contributions}

ASL, DL and MLH conceived and designed the study. ASL, DL and MLH conducted the data search. ASL and DMS conducted the title and abstract screeening, full text screeening and data extraction. ASL and DMS analysed the data and conducted the quality assessments. ASL wrote the first draft of the manuscript. ASL, DMS, MLH and DL assessed the confidence of the review findings. MLH, DL and DMS contributed to the interpretation of the data and review of the manuscript for intellectual content. All the authors have read and approved the manuscript for submission.

\section{References}

1. Organization, W.H., Increasing access to health workers in remote and rural areas through improved retention: global policy recommendations. 2010: World Health Organization.

2. Cabieses, B. and P. Bird, Glossary of access to health care and related concepts for low-and middle-income countries (LMICs): a critical review of international literature. International Journal of Health Services, 2014. 44(4): p. 845-861. 
3. Graves, B.A., Rural healthcare access: Issues for consideration in rural health research. Online journal of rural nursing and health care, 2012. 8(2): p. 2-4.

4. Sulemana, A. and R.D. Dinye, Access to healthcare in rural communities in Ghana: a study of some selected communities in the Pru District. European Journal of Research in Social Sciences, 2014. 2(4).

5. Zihindula, G., et al., A review on the contributions of NGOs in addressing the shortage of healthcare professionals in rural South Africa. Cogent Social Sciences, 2019. 5(1): p. 1674100.

6. Akinfaderin-Agarau, F., et al., Opportunities and limitations for using new media and mobile phones to expand access to sexual and reproductive health information and services for adolescent girls and young women in six Nigerian states. African journal of reproductive health, 2012. 16(2): p. 219-230.

7. Chandra-Mouli, V., et al., Contraception for adolescents in low and middle income countries: needs, barriers, and access. Reproductive health, 2014. 11(1): p. 1-8.

8. WHO, Assessment of barriers to accessing health services for disadvantaged adolescents in Tanzania. Brazzaville: WHO Regional Office for Africa; 2019.. Licence: CC BY-NC-SA 3.0 IGO. , 2019.

9. Ippoliti, N.B., G. Nanda, and R. Wilcher, Meeting the reproductive health needs of female key populations affected by HIV in low-and middle-income countries: a review of the evidence. Studies in family planning, 2017. 48(2): p. 121-151.

10. Hall, K.S., et al., Bad girl and unmet family planning need among Sub-Saharan African adolescents: the role of sexual and reproductive health stigma. Qualitative research in medicine \& healthcare, 2018. 2(1): p. 55.

11. Desrosiers, A., et al., A systematic review of sexual and reproductive health interventions for young people in humanitarian and lowerand-middle-income country settings. BMC public health, 2020. 20: p. 1-21.

12. Watkins, J.O.T.A., et al., Mobile phone use among patients and health workers to enhance primary healthcare: $A$ qualitative study in rural South Africa. Social Science \& Medicine, 2018. 198: p. 139-147.

13. Mechael, P.N., The case for mHealth in developing countries. Innovations: Technology, Governance, Globalization, 2009. 4(1): p. 103118.

14. Akter, S. and P. Ray, mHealth-an ultimate platform to serve the unserved. Yearbook of medical informatics, 2010. 19(01): p. 94-100.

15. Aranda-Jan, C.B., N. Mohutsiwa-Dibe, and S. Loukanova, Systematic review on what works, what does not work and why of implementation of mobile health (mHealth) projects in Africa. BMC public health, 2014. 14(1): p. 1-15.

16. Feroz, A., R. Jabeen, and S. Saleem, Using mobile phones to improve community health workers performance in low-and-middle-income countries. BMC Public Health, 2020. 20(1): p. 1-6.

17. Feroz, A.S., et al., Using mobile phones to improve young people sexual and reproductive health in low and middle-income countries: a systematic review to identify barriers, facilitators, and range of mHealth solutions. Reproductive Health, 2021. 18(1): p. 1-13.

18. Griffiths, F., et al., Mobile consulting (mConsulting) and its potential for providing access to quality healthcare for populations living in low-resource settings of low-and middle-income countries. Digital health, 2020. 6: p. 2055207620919594.

19. Hall, C.S., et al., Assessing the impact of mHealth interventions in low-and middle-income countries-what has been shown to work? Global health action, 2014. 7(1): p. 25606.

20. Organization, W.H., WHO guideline: recommendations on digital interventions for health system strengthening: web supplement 2: summary of findings and GRADE tables. 2019, World Health Organization.

21. Gottschalk, L.B. and N. Ortayli, Interventions to improve adolescents' contraceptive behaviors in low-and middle-income countries: a review of the evidence base. Contraception, 2014. 90(3): p. 211-225.

22. Mwaisaka, J., et al., Young people's experiences using an on-demand mobile health sexual and reproductive health text message intervention in Kenya: qualitative study. JMIR mHealth and uHealth, 2021. 9(1): p. e19109.

23. Sondaal, S.F.V., et al., Assessing the effect of $m$ Health interventions in improving maternal and neonatal care in low-and middle-income countries: a systematic review. Plos one, 2016. 11(5): p. e0154664.

24. Ippoliti, N.B. and K. L'Engle, Meet us on the phone: mobile phone programs for adolescent sexual and reproductive health in low-tomiddle income countries. Reproductive health, 2017. 14(1): p. 1-8.

25. Jennings, L., et al., Exploring the use of mobile phone technology for the enhancement of the prevention of mother-to-child transmission of HIV program in Nyanza, Kenya: a qualitative study. BMC public health, 2013. 13(1): p. 1-9.

26. Free, C., et al., The effectiveness of mobile-health technologies to improve health care service delivery processes: a systematic review and meta-analysis. PLoS Med, 2013. 10(1): p. e1001363. 
27. Moher, D., et al., Preferred reporting items for systematic reviews and meta-analyses: the PRISMA statement. PLoS med, 2009. 6(7): p. e1000097.

28. Frandsen, T.F., F.A. Gildberg, and E.B. Tingleff, Searching for qualitative health research required several databases and alternative search strategies: a study of coverage in bibliographic databases. Journal of clinical epidemiology, 2019. 114: p. 118-124.

29. Istepanian, R.S., Guest editorial special issue on mobile telemedicine and telehealth systems. IEEE Transactions on Information Technology in Biomedicine, 2000. 4(3): p. 194-194.

30. Moher, D., et al., Preferred reporting items for systematic review and meta-analysis protocols (PRISMA-P) 2015 statement. Systematic reviews, 2015. 4(1): p. 1-9.

31. Xiehang, C.D.L., Evaluation and prospect of reference management software--a case study of EndNote and NoteExpress. New Technology of Library and Information Service, 2009: p. Z1.

32. Serajuddin, U. and N. Hamadeh, New World Bank country classifications by income level: 2020-2021. World Bank Blogs, 2020.

33. Pluye, P., et al., A scoring system for appraising mixed methods research, and concomitantly appraising qualitative, quantitative and mixed methods primary studies in mixed studies reviews. International journal of nursing studies, 2009. 46(4): p. 529-546.

34. Jahangir, Y.T., et al., Provider Perspectives on Sexual Health Services Used by Bangladeshi Women with mHealth Digital Approach: A Qualitative Study. International Journal of Environmental Research and Public Health, 2020. 17(17): p. 6195.

35. Peprah, P., et al., Lessening barriers to healthcare in rural Ghana: providers and users' perspectives on the role of mHealth technology. A qualitative exploration. BMC medical informatics and decision making, 2020. 20(1): p. 27.

36. Braun, R., et al., An evaluation of a family planning mobile job aid for community health workers in Tanzania. Contraception, 2016. 94(1): p. 27-33.

37. Dev, R., et al., Acceptability, feasibility and utility of a Mobile health family planning decision aid for postpartum women in Kenya. Reproductive health, 2019. 16(1): p. 1-11.

38. Logie, C., et al., Sexual and reproductive health mobile apps: results from a cross-sectional values and preferences survey to inform World Health Organization normative guidance on self-care interventions. Global Health Action, 2020. 13(1): p. 1796346.

39. Ibembe, J.D., Mobile phone use and reproductive health care in Nakuru Provincial Hospital, Kenya. 2011.

40. Ong, K.K.X., et al., Perceived barriers and facilitators in using text and voice messaging for improving HIV and sexual and reproductive health of female entertainment workers in Cambodia: a qualitative study. Mhealth, 2020. 6.

41. Khatun, F., et al., Community readiness for adopting $m$ Health in rural Bangladesh: a qualitative exploration. International journal of medical informatics, 2016. 93: p. 49-56.

42. Hirsch-Moverman, Y., et al., Using mHealth for HIV/TB treatment support in Lesotho: enhancing patient-provider communication in the START study. Journal of acquired immune deficiency syndromes (1999), 2017. 74(Suppl 1): p. S37.

43. Hampshire, K., et al., Who bears the cost of 'informal mhealth'? Health-workers' mobile phone practices and associated political-moral economies of care in Ghana and Malawi. Health policy and planning, 2017. 32(1): p. 34-42.

44. Chang, L.W., et al., Impact of a mHealth intervention for peer health workers on AIDS care in rural Uganda: a mixed methods evaluation of a cluster-randomized trial. AIDS and Behavior, 2011. 15(8): p. 1776-1784.

45. Amoakoh-Coleman, M., et al., Effectiveness of mHealth interventions targeting health care workers to improve pregnancy outcomes in low-and middle-income countries: a systematic review. Journal of medical Internet research, 2016. 18(8): p. e226.

46. NOVEL, U.A., L.-C. NON-INVASIVE, and M. IMAGER, MONDAY, MAY 19, 2014. Concurrent Oral Presentations Abstract Index, 2014 : p. 37.

47. Kazi, A.M., et al., Assessing mobile phone access and perceptions for texting-based mHealth interventions among expectant mothers and child caregivers in remote regions of northern Kenya: a survey-based descriptive study. JMIR public health and surveillance, 2017. 3(1): p. e5386.

48. Parajuli, R. and P. Doneys, Exploring the role of telemedicine in improving access to healthcare services by women and girls in rural Nepal. Telematics and Informatics, 2017. 34(7): p. 1166-1176.

49. Adeagbo, O., et al., Exploring people's candidacy for mobile health-supported HIV testing and care services in rural KwaZulu-Natal, South Africa: Qualitative study. Journal of medical Internet research, 2019. 21(11): p. e15681.

50. Duclos, V., et al., Situating mobile health: a qualitative study of mHealth expectations in the rural health district of Nouna, Burkina Faso. Health research policy and systems, 2017. 15(1): p. 47.

51. Organization, W.H., Task shifting: rational redistribution of tasks among health workforce teams: global recommendations and guidelines. 2007. 
52. Smith, S., et al., Task-shifting and prioritization: a situational analysis examining the role and experiences of community health workers in Malawi. Human Resources for Health, 2014. 12(1): p. 1-13.

53. Ahmed, T., et al., E-health and M-Health in Bangladesh: Opportunities and Challenges. 2014.

54. Achampong, E.K., The state of information and communication technology and health informatics in Ghana. Online journal of public health informatics, 2012. 4(2).

55. Willcox, M., K. Romano, and D. Hutchful, Mobile Technology for Community Health (MOTECH) in Ghana: is maternal messaging and provider use of technology cost effective in improving maternal and child health outcomes at scale?

56. Kamanga, A., et al., Rural health centres, communities and malaria case detection in Zambia using mobile telephones: a means to detect potential reservoirs of infection in unstable transmission conditions. Malaria journal, 2010. 9(1): p. 1-7.

57. Long, L.-A., G. Pariyo, and K. Kallander, Digital technologies for health workforce development in low-and middle-income countries: a scoping review. Global Health: Science and Practice, 2018. 6(Supplement 1): p. S41-S48.

58. Early, J., et al., Use of mobile health (mHealth) technologies and interventions among community health workers globally: a scoping review. Health promotion practice, 2019. 20(6): p. 805-817.

59. Kruse, C., et al., Barriers to the use of mobile health in improving health outcomes in developing countries: systematic review. Journal of medical Internet research, 2019. 21(10): p. e13263.

60. Laar, A., et al., Assessment of mobile health technology for maternal and child health services in rural Upper West Region of Ghana. Public health, 2019. 168: p. 1-8.

61. Firestone, C., The Mobile Generation: Global Transformations at the Cellular Level. 2007: Aspen Institute.

62. Källander, K., et al., Mobile health (mHealth) approaches and lessons for increased performance and retention of community health workers in low-and middle-income countries: a review. Journal of medical Internet research, 2013. 15(1): p. e17.

\section{Figures}




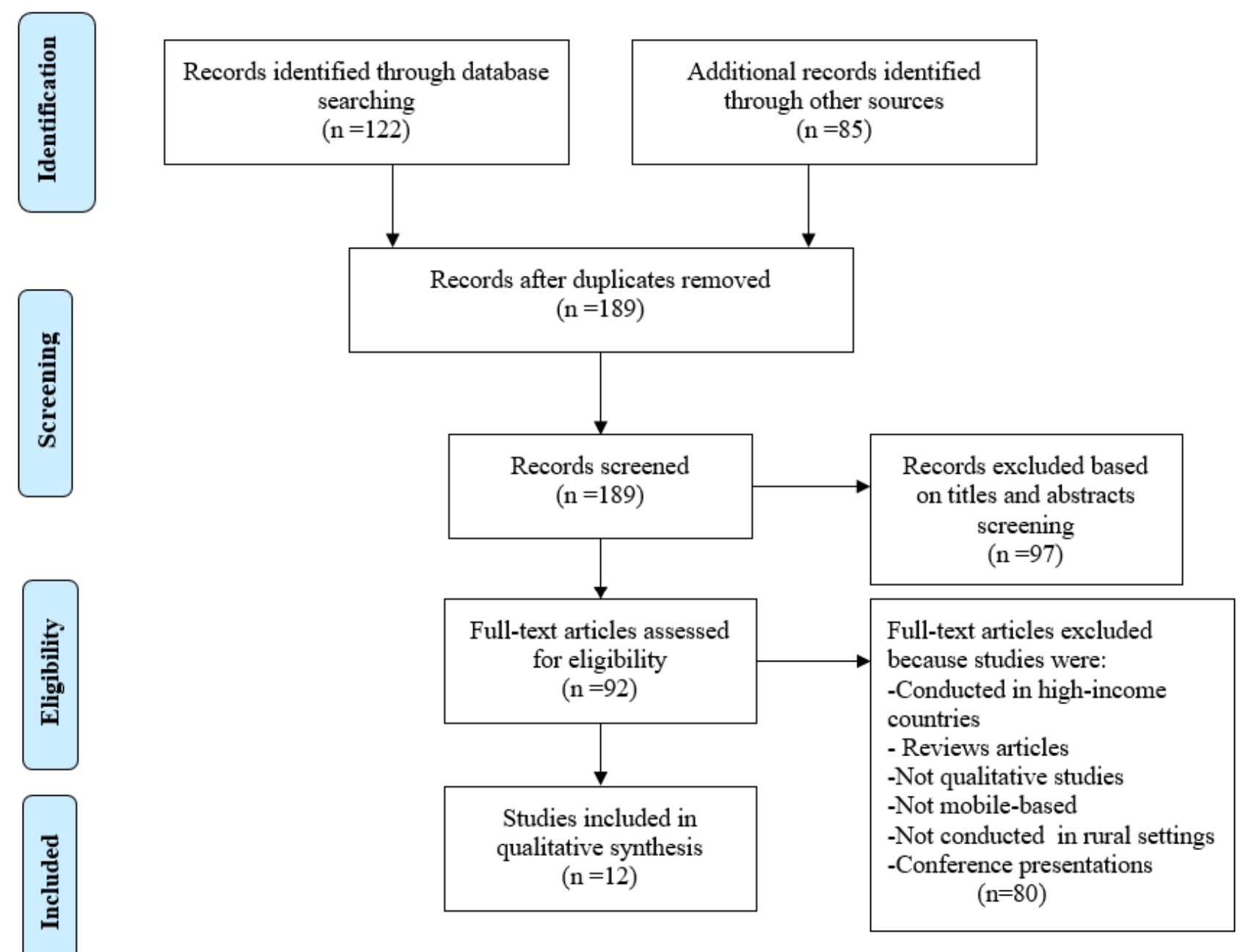

Figure 1

Results of PRISMA Flow diagram

\section{Supplementary Files}

This is a list of supplementary files associated with this preprint. Click to download.

- OnlineSupplement.docx 